\title{
Translucency and color stability of resin composite and dental adhesives as modeling liquids - A one-year evaluation
}

\begin{abstract}
José Augusto SEDREZ-PORTO(a) Eliseu Aldrighi MÜNCHOW(b) Maximiliano Sérgio $\mathrm{CENCI}^{(\mathrm{a})}$ Tatiana PEREIRA-CENCI(a)
\end{abstract}

(a) Universidade Federal de Pelotas - UFPel, School of Dentistry, Graduate Program in Dentistry, Pelotas, RS, Brazil.

(b) Universidade Federal de Juiz de Fora - UFJF, Health Science Institute, Department of Dentistry, Governador Valadares, MG, Brazil.

Declaration of Interest: The authors certify that they have no commercial or associative interest that represents a conflict of interest in connection with the manuscript.

Corresponding Author:

Tatiana Pereira-Cenci

E-mail: tatiana.dds@gmail.com

htps://doi.org/10.1590/1807-3107BOR-2017.vol31.0054

Submitted: Nov 30, 2016

Accepted for publication: May 05, 2017

Last revision: May 22, 2017
Abstract: The aim of this study is to evaluate the influence of modeling liquids on the translucency and color shade of resin composites (RCs) after one year of storage. RC specimens were prepared using either a conventional insertion technique (control; without modeling liquid) or a restorative dental modeling insertion technique (RDMIT) with dental adhesives as modeling liquids (Scotchbond ${ }^{\mathrm{TM}}$ Multi-Purpose [SBMP; 3M ESPE] or AdperTM Single Bond 2 [SB; 3M ESPE]). The initial colors of the specimens were obtained with a digital spectrophotometer and the CIEL ${ }^{*} a^{*} b^{*}$ color system, after which specimens were stored $\left(37^{\circ} \mathrm{C}\right)$ in distilled water or red wine for 12 months. Color measurements were reassessed after 6 and 12 months of storage, and scanning electron microscopy was performed after 12 months. Translucency and color change $\left(\Delta E^{*}\right)$ were calculated and analyzed using ANOVA and Tukey's test $(\alpha=5 \%)$. RC samples prepared via RDMIT showed a translucency similar to that of control samples. $\Delta E^{*}$ was also less intense for RCs containing SBMP than for RCs containing SB. Specimens stored in wine showed a clear pattern of degradation, especially in the control group, and surface degradation seemed to be less intense for specimens prepared with SBMP and SB than for specimens without. Specimens stored in water did not show clear evidence of surface degradation. RDMIT appears to be an interesting approach to reduce $\Delta E^{*}$ in RCs over time without negative effects on the translucency of the material. However, the modeling liquid should feature a hydrophobic composition, similar to that used in the SBMP group, the achieve the best results.

Keywords: Aging; Dental Materials; Microscopy, Electron, Scanning.

\section{Introduction}

The restorative dental modeling insertion technique (RDMIT) proposed for esthetic restorations has aroused growing interest among dental practitioners. In RDMIT, low viscosity, resin-based solutions are used to facilitate resin composite (RC) sculpture and manipulation, especially in the case of large restorations, which usually require a combination of composites with different optical characteristics (e.g., translucency, shade, chrome value) in an attempt to mimic the properties of natural teeth..$^{1,2}$ The modeling resins may be applied directly to the composite increments with a brush or used as lubricants in the insertion instruments; both strategies improve 
the ease of sculpting aesthetic restorations compared with the conventional technique, i.e., without modeling liquids. Nevertheless, concerns regarding the effects of RDMIT on the mechanical behavior and physical stability of composite restorations have increased, and a growing number of researchers have sought to evaluate RCs prepared via RDMIT.

Barcellos et al. ${ }^{2}$ investigated the influence of different modeling liquids, such as composite wetting resins or dental adhesives, on the cohesive strength of the resulting composites; the authors showed that RDMIT improved the cohesive bond strength of composite interfaces compared with specimens prepared without modeling liquid. In addition, the modeling liquid that produced the highest cohesive strength was the solvent-free Scotchbond ${ }^{\mathrm{TM}}$ Multi-Purpose Adhesive (SBMP; 3M ESPE); other compositions containing solvents in their formulation yielded significantly lower cohesiveness. In another study, ${ }^{3}$ the use of SBMP, a hydrophobic unfilled resin, as a modeling liquid also enhanced the mechanical properties and stability of the resulting composites; the same trend was not observed in specimens prepared using a more hydrophilic composition. Thus, RDMIT apparently contributes to improving the characteristics of the composites, but this improvement depends on the type of modeling liquid used.

The optical appearance of composite restorations must ideally not change over time. In a study by Tuncer et al., ${ }^{4}$ the color stability of RCs was negatively affected by covering with a superficial layer of modeling resin. However, when present between layers of $\mathrm{RC}$, modeling liquids are able to reduce or delay staining in the material. ${ }^{3,5}$ The optical properties of RCs are not expected to remain stable over time, especially considering the degradation phenomena that composites may undergo after placement in the oral environment. ${ }^{6,78}$ Among these properties, color shade and translucency are the main characteristics susceptible to modification, and special attention must be given to restorations prepared using modeling liquids because these liquids may influence the color and translucency of the composites after aging.

The analysis of color and its perception is another fundamental aspect determining the clinical success of the restorative procedure. According to some studies, ${ }^{9,10}$ professionals may suffer bias during color evaluation since color perceptions may change over time due to both external (e.g., light throughout the day) and individual (e.g., color sense in different moments of life) variables. Thus, color evaluations using mechanical instruments may enable color analysis with reduced risk of bias or human fatigue, introduce a standardized method of evaluating color parameters, and increase the success of the restorative treatment.

The purpose of this study is to investigate the influence of modeling liquid on the translucency and color shade of RCs after one year of storage. Here, the null hypothesis is that composites prepared via RDMIT would present a translucency and color alteration similar to those of composites prepared using the conventional insertion technique after aging.

\section{Methodology}

This in vitro $3 \times 2 \times 2$ factorial study $(n=5)$ evaluated three distinct factors: presence of the modeling liquid, storage solution, and storage period. In total, $30 \mathrm{RC}$ specimens (Filtek ${ }^{\mathrm{TM}}$ Z350 XT, 3M ESPE, St. Paul, $\mathrm{MN}, \mathrm{USA}$ ) with or without a modeling liquid (resin adhesive, i.e., SBMP [the bond component of Adper ${ }^{\mathrm{TM}}$ Scotchbond ${ }^{\mathrm{TM}}$ Multi-Purpose Adhesive, 3M ESPE] or SB [Adper ${ }^{\mathrm{TM}}$ Single Bond 2 Adhesive, 3M ESPE]) were prepared to investigate the translucency and color stability of the resulting composites over time.

\section{Specimen preparation and group allocation}

Each specimen was prepared by placing four increments of $\mathrm{RC}$ into a silicone mold (6 mm diameter $\times 2 \mathrm{~mm}$ thickness) as described elsewhere. ${ }^{3}$ For specimens prepared without the modeling liquid (control), four composite increments (approximate thickness, $0.5 \mathrm{~mm}$ ) were packed into the mold and covered with a mylar strip; light-activation was then performed for $20 \mathrm{~s}$ on both top and bottom surfaces using a light-emitting diode (LED, Radii ${ }^{\circledR}$, Bayswater, VIC, Australia; irradiance, $900 \mathrm{~mW} / \mathrm{cm}^{2}$ ) curing unit. Any excess material was gently removed using a scalpel. For specimens prepared with the modeling liquid, after placement of the first composite increment, SBMP or SB was applied on the composite surface with a disposable 
brush (Microbrush ${ }^{\circledast}$ International, Grafton, WI, USA); a new increment was then placed, modeled, and coated with another pellicle of the modeling liquid until the fourth increment was finally placed and modeled. Mylar strips were then placed over the surface of the specimens, and light-activation was performed as described above with the LED. Any excess was also gently removed using a scalpel. After light-activation, all specimens were polished with medium, fine, and extra-fine aluminum oxide abrasive disks (Sof-Lex Pop On Orange series, 3M ESPE) for $15 \mathrm{~s}$ per disk and then with a felt disk containing extra-fine $(2-4 \mu \mathrm{m})$ diamond paste (Diamond AC II, FGM, Joinville, SC, Brazil). ${ }^{3}$

After polishing, the specimens were randomly allocated into two groups ( $n=5)$ according to the storage media: distilled water ( $\mathrm{pH}$ 5.9) or red wine (Cabernet Sauvignon 2007, Concha y Toro, Las Condes, Chile - pH 3.6 and 14.5 vol.\% alcohol). The media were renewed weekly, and specimens were kept immersed at $37^{\circ} \mathrm{C}$ for 12 months.

\section{Translucency and color stability tests}

Translucency and color tests were performed following ISO/TR 28642:2016 standards. From the first to the last measurement procedure, specimens were tested under the same environmental conditions, i.e., similar light and humidity.

Specimen translucency was measured using the translucency parameter (TP) method. ${ }^{11}$ The color parameters of each specimen were recorded according to the CIE $L^{*} a^{*} b^{*}$ system ( $L^{*}$ : white/black; $a^{*}$ : red/green; $b^{*}$ : yellow/blue $)^{12}$ against white $\left(L_{\mathrm{W}}^{*}=94.44, a_{\mathrm{W}}^{*}=0.26\right.$, $\left.b^{*}{ }_{\mathrm{W}}=1.69\right)$ and black $\left(L_{\mathrm{B}}^{*}=1.38, a_{\mathrm{B}}^{*}=0.00, b_{\mathrm{B}}^{*}=0.06\right)$ Munsell-like neutral-value scale sheet backgrounds (AG-5330, BYK Gardner, USA). Measurements were obtained using a digital spectrophotometer (Vita Easyshade, Vita Zahnfabrik, Bad Sackingen, Germany), and the TP of all specimens was evaluated at different time periods: immediately after polishing (baseline) and after 6 and 12 months of storage in each medium. TP was then calculated using the following formula: ${ }^{12}$

$$
T P=\sqrt{\left(L_{W}^{*}-L_{B}^{*}\right)^{2}+\left(a_{W}^{*}-a_{B}^{*}\right)^{2}+\left(b_{W}^{*}-b_{B}^{*}\right)^{2}}
$$

where $L^{*} W, a^{*} W$, and $b^{*} W$ are measurements over the white background and $L^{*} B, a^{*} B$, and $b^{*} B$ are measurements over the black background.
The color stability of specimens was evaluated using the color parameters measured over a white background in the translucency test. Color change $\left(\Delta E^{*}\right)$ was calculated according to the following formula: ${ }^{12}$

$$
\Delta E^{*}=\sqrt{\left(\Delta L^{*}\right)^{2}+\left(\Delta a^{*}\right)^{2}+\left(\Delta b^{*}\right)^{2}}
$$

where $\Delta L^{*}, \Delta a^{*}$, and $\Delta b^{*}$ are the differences between the final and initial $L^{*}, a^{*}$, and $b^{*}$ color parameters, respectively. $\Delta E^{*}$ was measured after 6 and 12 months of storage in each medium.

\section{Scanning electron microscopy (SEM) analysis}

After 12 months of storage, specimens from each group were randomly selected for surface evaluation by SEM. Specimens were dried in a desiccator at $37^{\circ} \mathrm{C}$, sputter-coated with gold/palladium, and then evaluated under a scanning electron microscope (SSX-550, Shimadzu, Tokyo, Japan). The obtained images were qualitatively analyzed.

\section{Statistical analysis}

All data were analyzed with the statistical program SigmaPlot 12 (Systat Software Inc., San Jose, USA). Two-way repeated-measures ANOVA and the Tukey test $(\alpha=5 \%)$ were used to analyze the effect of factors "presence of modeling liquid" and "storage period" on the TP and $\Delta E^{*}$ of groups investigated. Specimens stored in water were not statistically compared with their counterparts stored in wine.

\section{Results}

The TP results are shown in Table 1 and Figure 1. Whereas the presence of modeling liquid was a significant factor only for specimens stored in distilled water $(p=0.012)$, the storage period was a significant factor for all specimens regardless of the storage solution tested ( $p=0.007)$. Factors did not statistically interact with each other $(p=0.573)$. Among specimens stored in distilled water, SBMP and SB showed higher TP than the control at baseline $(p=0.036)$ but similar values after 6 and 12 months of storage $(p=0.065)$. TP increased in the control and SB groups after 6 months $(p=0.049)$, although no further increase was observed after 12 months of water storage $(p=0.959)$. SBMP presented a stable 
Table 1. Translucency parameter means and standard deviations $( \pm \mathrm{SD})$ of groups after different periods of water or wine storage.

\begin{tabular}{|c|c|c|c|c|c|c|}
\hline \multirow{3}{*}{ Groups } & \multicolumn{6}{|c|}{ Storage condition } \\
\hline & \multicolumn{3}{|c|}{ Distilled water } & \multicolumn{3}{|c|}{ Red wine } \\
\hline & Baseline & 6 months & 12 months & Baseline & 6 months & 12 months \\
\hline Control & $5.5 \pm 1.4^{\mathrm{B}, \mathrm{b}}$ & $8.9 \pm 2.8^{A, a}$ & $9.0 \pm 0.7^{A, a}$ & $7.1 \pm 1.3^{A, a}$ & $8.5 \pm 1.3^{A, a}$ & $9.0 \pm 1.3^{A, a}$ \\
\hline SBMP & $8.4 \pm 2.1^{A, a}$ & $9.9 \pm 1.9 \mathrm{~A}, \mathrm{a}$ & $10.2 \pm 1.2^{\mathrm{A}, \mathrm{a}}$ & $9.2 \pm 1.7^{\mathrm{A}, \mathrm{a}}$ & $9.2 \pm 1.6^{\mathrm{A}, \mathrm{a}}$ & $10.8 \pm 2.8^{A, a}$ \\
\hline SB & $8.8 \pm 1.7^{\mathrm{A}, \mathrm{b}}$ & $11.5 \pm 2.4^{\mathrm{A}, \mathrm{a}}$ & $10.5 \pm 0.5^{\mathrm{A}, \mathrm{ab}}$ & $9.4 \pm 0.5^{\mathrm{A}, \mathrm{b}}$ & $11.5 \pm 2.9^{\mathrm{A}, \mathrm{ab}}$ & $12.4 \pm 3.6^{A, a}$ \\
\hline
\end{tabular}

Different superscript uppercase and lowercase letters respectively indicate statistical significant differences among groups (columns) and between time points (rows) $(p<0.05)$.
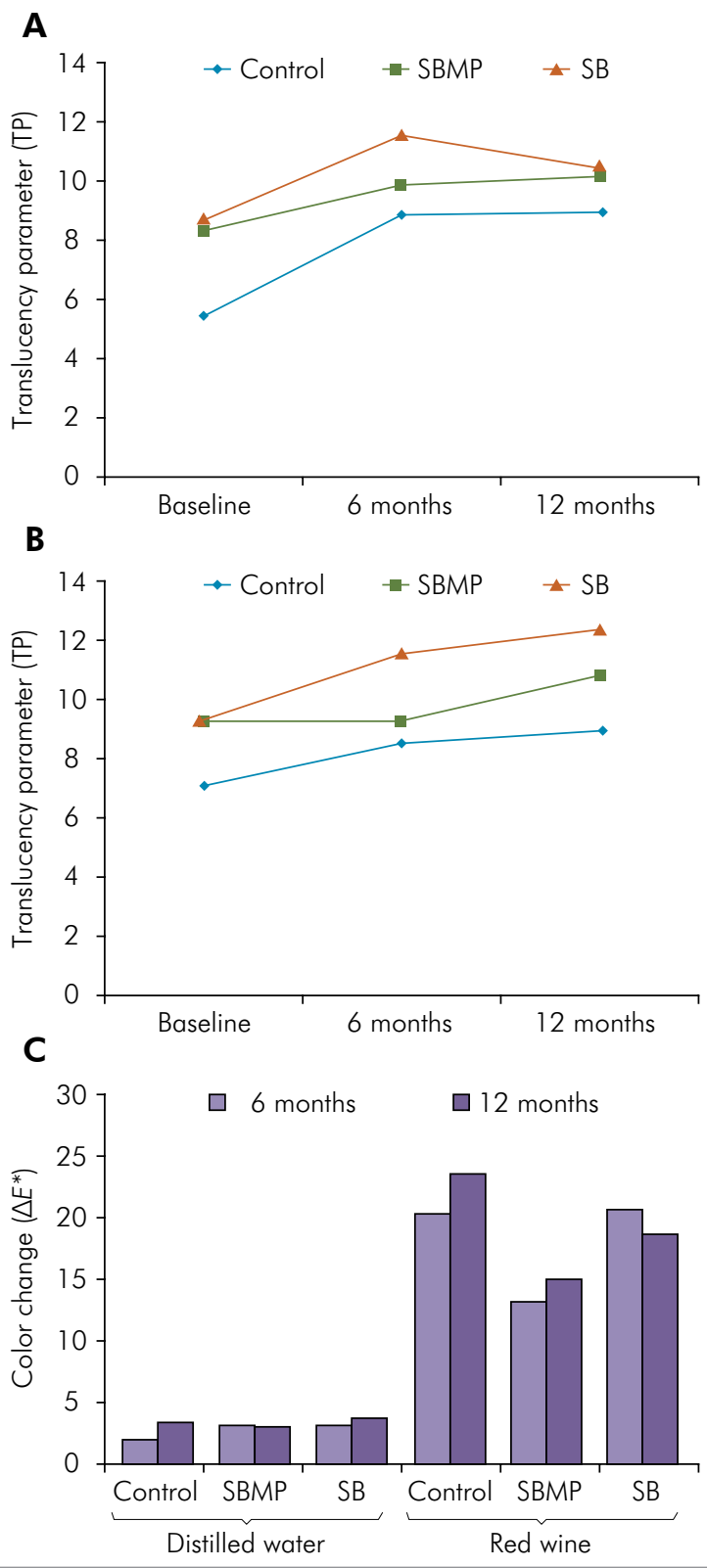

Figure 1. Translucency parameter (TP) of groups at different time points after immersion in distilled water (a) or red wine (b). Color change $\left(\Delta E^{*}\right)$ of groups after 6 or 12 months of immersion in distilled water or red wine storage (c).
TP over time. Among specimens stored in red wine, in general, the TP of the groups did not differ from each other $(p=0.060)$, although SB showed a higher TP value compared with the baseline after 12 months of storage ( $p=0.037)$.

$\Delta E^{*}$ results are shown in Table 2 and Figure 1. Whereas the presence of the modeling liquid was a significant factor only for specimens stored in red wine $(\mathrm{p}=0.002)$, the storage period was a significant factor for all specimens stored in distilled water $(p=0.008)$. Statistically significant interactions were not observed between the factors tested, regardless of the storage solution $(p=0.085)$. Among specimens stored in distilled water, the $\Delta E^{*}$ of the control was the greatest after 12 months, and specimens containing the modeling liquid showed color stability over time $(p=0.079)$. Among specimens stored in red wine, SBMP presented a lower $\Delta E^{*}$ than the control after 6 and 12 months of storage $(\mathrm{p}=0.007)$, while $\mathrm{SB}$ showed a $\Delta E^{*}$ similar to that of the control $(\mathrm{p}=0.071)$. Comparing the SBMP and SB groups, SB showed greater $\Delta E^{*}$ than SBMP after 6 months of storage $(p=0.004)$, but similar results between groups were observed after 12 months ( $\mathrm{p}=0.161)$.

Figure 2 shows SEM images of the specimens prepared with or without the modeling liquid and stored in distilled water or red wine after 12 months. Specimens stored in wine presented a clear pattern of degradation, especially for the control group. Surface degradation seemed to be less intense for specimens prepared with SBMP and SB than for control specimens, and SB composites were associated with a generally rougher topography than SBMP samples. Specimens stored in water did not show clear evidence of surface degradation. 
Table 2. Color change means and standard deviations $( \pm S D)$ of groups after 6 and 12 months of water or wine storage.

\begin{tabular}{|c|c|c|c|c|}
\hline \multirow{3}{*}{ Groups } & \multicolumn{4}{|c|}{ Storage condition } \\
\hline & \multicolumn{2}{|c|}{ Distilled water } & \multicolumn{2}{|c|}{ Red wine } \\
\hline & 6 months & 12 months & 6 months & 12 months \\
\hline Control & $2.0 \pm 0.2^{\mathrm{A}, \mathrm{b}}$ & $3.4 \pm 0.3^{A, a}$ & $20.2 \pm 1.3^{A, a}$ & $23.4 \pm 2.9^{A, a}$ \\
\hline SBMP & $3.1 \pm 0.8^{A, a}$ & $3.1 \pm 0.8^{A, a}$ & $13.2 \pm 2.0^{B, a}$ & $14.8 \pm 2.5^{\mathrm{B}, \mathrm{a}}$ \\
\hline SB & $3.1 \pm 0.6^{A, a}$ & $3.7 \pm 0.7^{A, a}$ & $20.6 \pm 5.4^{A, a}$ & $18.6 \pm 3.6^{A B, a}$ \\
\hline
\end{tabular}

Different superscript uppercase and lowercase letters respectively indicate statistical significant differences among groups (columns) and between time points (rows) $(p<0.05)$.

\section{Discussion}

Minimal $\Delta E^{*}$ is one of the major characteristics desired for RC restorations, especially when involving anterior teeth. However, composites commonly suffer from degradation because of their polymeric nature, which results in a compromised color appearance over time. According to recent studies, ${ }^{3,5}$ the use of modeling liquids (e.g., unfilled adhesive resins) between RC layers may be a useful strategy to reduce or delay composite staining. Thus, the purpose of the present study is to investigate whether the translucency and color shade of RCs prepared with modeling liquids would change after one year of storage. In the overall analysis, the translucency and $\Delta E^{*}$ of a composite depended on the type of modeling liquid used.

Translucency and color shade are two optical properties that vary according to the composition of the materials tested. In the present work, refractive index mismatches between the modeling liquid (unfilled resin) and composite (highly filled resin) may be expected to affect the way light is transmitted within materials containing the former, ${ }^{13,14,15}$ thereby changing the translucency and opacity of the resulting composites. This expectation was confirmed at baseline, in which specimens containing SBMP or SB showed $29 \%-60 \%$ higher TP than the control, (Figures 1a, 1b). This change may be explained by possible reductions in light scattering in specimens prepared with the modeling liquid, since unfilled resins could facilitate light transmission within the material ${ }^{14}$ and show an increase in translucency. The TP of SBMP samples remained stable over time regardless of the storage medium; by contrast, the TP of SB samples increased after 6 months of water storage and 12 months of wine storage compared with the baseline values. These findings demonstrate that the type of modeling liquid used for layering plays an important role on the translucency of the composite. While SBMP exhibits a moderately hydrophobic composition, SB contains a larger amount of solvents, which make it fairly hydrophilic. In a study by Münchow et al., ${ }^{3}$ specimens containing SB showed increased translucency after one day of water storage, and the authors proposed a series of events to explain this result: First, the solvent molecules of SB were entrapped between increments of the composite during polymerization. Then, after some period of water immersion, the solvent was eliminated from the bulk of the material, leading to free spaces that could be filled with water. This process facilitates light propagation, which increases the composite's translucency.

Similar to specimens prepared with SB, control specimens demonstrated increased TP after 6 months of water storage, which may be explained by the strong tendency of polymer-based materials to undergo hydrolysis. ${ }^{8}$ Indeed, water is a potent solvent capable of degrading the intermolecular bonds of RCs. ${ }^{16}$ The presence of SBMP within the composite helped keep its TP stable over time, even after one year of water or wine storage, likely because the modeling liquid exerts a protective effect against hydrolysis. This finding is corroborated by recent studies. ${ }^{3,5}$ Specimens prepared with SBMP appeared to be stronger and more cohesively packed than those prepared without, and these features could positively influence the chemophysical stability of the composite. ${ }^{2,3}$ The absence of translucency changes for specimens stored in wine, with the only exception occurring in the SB group after 12 months of storage, is notable. Wine is a potent staining medium with a low $\mathrm{pH}$ and moderately high alcoholic content compared with distilled water; these characteristics promote a more aggressive change 
in the optical properties of an RC specimen. Wine stains may be able to equally impregnate the surface of specimens, regardless of the presence or absence of modeling liquids, ${ }^{2}$ and this supposition could explain the similar TP values observed among the groups. Despite these initial results, only specimens containing SB showed less translucency after 12 months of storage, once again confirming that the presence of hydrophilic molecules within the composite may compromise translucency stability over time.

Hydrolytic degradation of composite restorations may affect not only translucency but also the color shade of the material. In the present study, $\Delta E^{*}$ was monitored after one year of water/wine storage. Specimens stored in water showed $\Delta E^{*}$ values lower than 3.3 after 6 months (Table 2); this change may be considered clinically acceptable since 3.3 is a threshold for visual perception of $\Delta E^{*} \cdot{ }^{17}$ At this limit, $\Delta E^{*}$ can be easily detected by laypersons. Here, we used the CIE $L^{*} a^{*} b^{*}$ system to evaluate $\Delta E^{*}$. Although some recent studies have demonstrated that another measurement system, i.e., CIEDE2000, may provide a better fit than the former in the evaluation of color difference thresholds, ${ }^{10,18,19,20}$ many other studies use the CIE L*a*b* color system to analyze color outcomes in dentistry ${ }^{3,5,21,22,23}$ because this system features predictability and clinical importance.
$\Delta E^{*}$ did not increase after 12 months of storage in specimens prepared with the modeling liquids. By contrast, control specimens exhibited higher $\Delta E^{*}$ after 12 months. Again, while hydrolysis is a common phenomenon that degrades composites and affects their optical properties, the presence of a modeling liquid may prevent the rapid degradation of the material and discoloration. Despite the absence of a statistically significant $\Delta E^{*}$ in the SB group after 12 months of water storage, the $\Delta E^{*}$ of this group was higher than 3.3 , which means the color perceived was clinically different from that at baseline. This finding bears importance because it demonstrates that less hydrophilic compositions, such as SBMP, are better options as modeling liquids for RC restorations than more hydrophilic ones.

$\Delta E^{*}$ was intense for specimens stored in red wine (Figure 1c), although no significant change among groups was seen after 6 months of storage. Indeed, wine is a potent staining solution; its high pigmentation potential, ${ }^{24}$ low $\mathrm{pH}$, and alcoholic content enable it to degrade composites by matrix decomposition and filler leakage. ${ }^{25}$ According to the present findings, specimens stored in wine demonstrated more surface degradation than specimens stored in water (Figure 2), thereby corroborating the $\Delta E^{*}$ results. Moreover, SBMP showed $53 \%$ and $58 \%$ less discoloration than the control

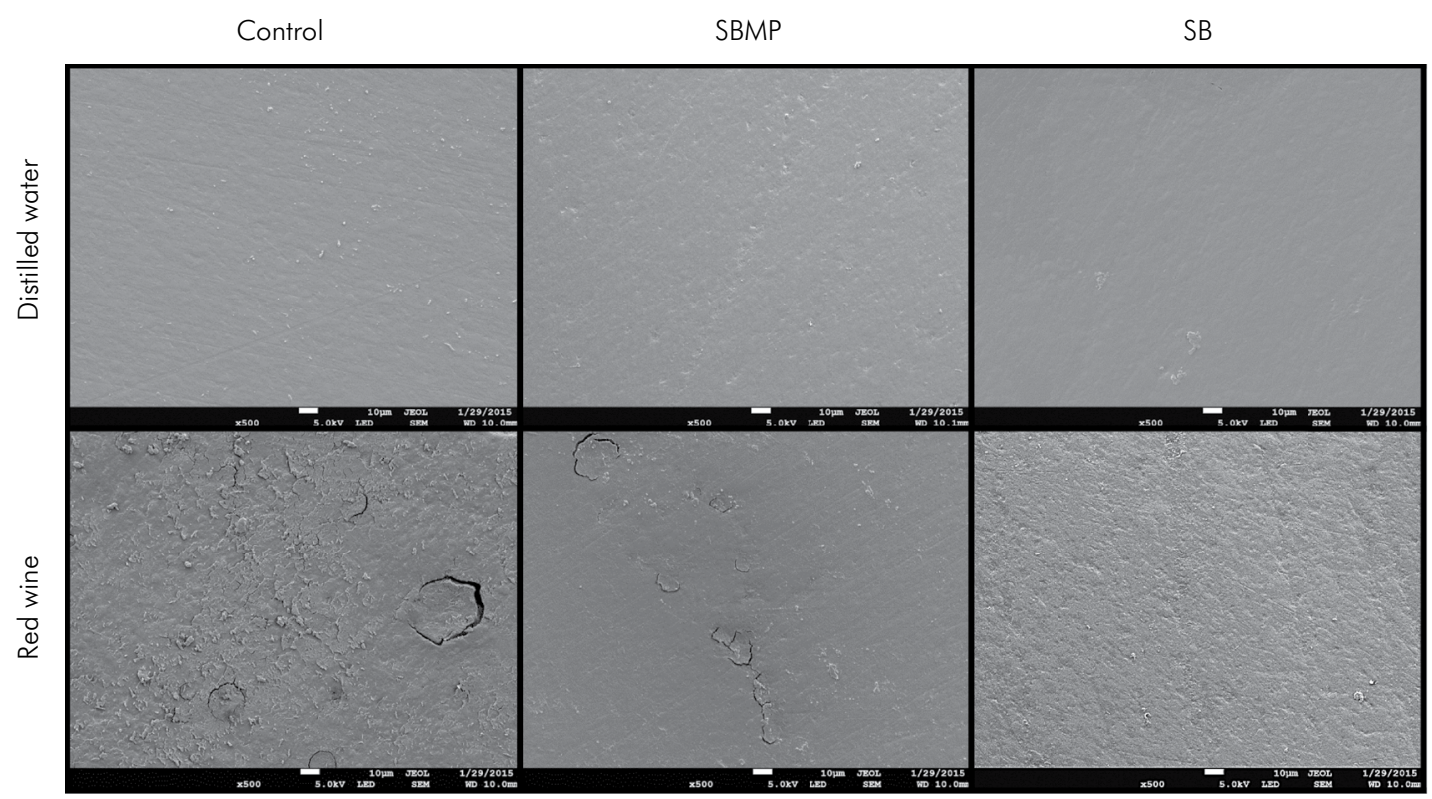

Figure 2. SEM micrographs showing the surface topography of specimens prepared with (SBMP and SB) or without (control) modeling liquid after 12 months of distilled water or red wine storage. 
after 6 and 12 months of storage, respectively, whereas SB and the control exhibited similar discoloration patterns (nearly $2 \%$ more and $26 \%$ less discoloration at 6 and 12 months, respectively). The hydrophobic composition of SBMP likely protected the polymerbased matrix of the composite from rapid staining. In a study by Münchow et al., ${ }^{3}$ specimens prepared with SB as a modeling liquid showed higher water sorption than specimens prepared with SBMP; this features enhances hydrolytic degradation within the bulk of the composite and pigmentation from the dye in the staining medium.

Taking all of the findings together, the null hypothesis tested here was accepted for translucency but rejected for color stability. While composites prepared following the proposed RDMIT showed a translucency similar to that of the control after aging, $\Delta E^{*}$ was less intense for composites containing SBMP than for those containing
SB. Although color measurement using a digital spectrophotometer may not comprise a real condition for evaluation, it may present a useful approach for quantifying color coordinates from distinct groups, thus allowing the proper control and monitoring of specimens submitted to aging processes.

\section{Conclusions}

The RDMIT procedure used in this study appears to be an interesting approach to reduce $\Delta E^{*}$ in RCs over time, at least for composites with compositions similar to that investigated here (Filtek ${ }^{\mathrm{TM}} \mathrm{Z} 350 \mathrm{XT}$ ). The proposed technique did not exert negative effects on the translucency of the material investigated. However, the modeling liquid should feature a hydrophobic composition, similar to that used in the SBMP group, to achieve the best effects.

\section{References}

1. Caughman WF, Comer RW, Zardiackas LD. Effects of diluents on physical properties of a light-cured composite Prisma-Fill. Oper Dent. 1984;9(3):82-5.

2. Barcellos DC, Pucci CR, Torres CR, Goto EH, Inocencio AC. Effects of resinous monomers used in restorative dental modeling on the cohesive strength of composite resin. J Adhes Dent. 2008;10(5):351-4.

3. Münchow EA, Sedrez-Porto JA, Piva E, Pereira-Cenci T, Cenci MS. Use of dental adhesives as modeler liquid of resin composites. Dent Mater. 2016;32(4):570-7. https://doi.org/10.1016/j.dental.2016.01.002

4. Tuncer S, Demirci M, Tiryaki M, Unlü N, Uysal O. The effect of a modeling resin and thermocycling on the surface hardness, roughness, and color of different resin composites. J Esthet Restor Dent. 2013;25(6):404-19. https://doi.org/10.1111/jerd.12063

5. Sedrez-Porto JA, Münchow EA, Brondani LP, Cenci MS, Pereira-Cenci T. Effects of modeling liquid/resin and polishing on the color change of resin composite. Braz Oral Res. 2016;30(1):e88. https://doi.org/10.1590/1807-3107BOR-2016.vol30.0088

6. Prodan DA, Gasparik C, Mada DC, Miclăuş V, Băciuţ M, Dudea D. Influence of opacity on the color stability of a nanocomposite. Clin Oral Investig. 2015;19(4):867-75. https://doi.org/10.1007/s00784-014-1315-1

7. Llena C, Fernandez S, Forner L. Color stability of nanohybrid resin-based composites, ormocers and compomers. Clin Oral Investig. 2017;21(4):1071-7. https://doi.org/10.1007/s00784-016-1850-z

8. Ferracane JL. Resin composite: state of the art. Dent Mater. 2011;27(1):29-38. https://doi.org/10.1016/i.dental.2010.10.020

9. Chu SJ, Trushkowsky RD, Paravina RD. Dental color matching instruments and systems. Review of clinical and research aspects. J Dent. 2010;38 Suppl 2:e2-16. https://doi.org/10.1016/i.jdent.2010.07.001

10. Pecho OE, Ghinea R, Alessandretti R, Pérez MM, Della Bona $A$. Visual and instrumental shade matching using CIELAB and CIEDE2000 color difference formulas. Dent Mater. 2016;32(1):82-92. https://doi.org/10.1016/j.dental.2015.10.015

11. Johnston WM, Ma T, Kienle BH. Translucency parameter of colorants for maxillofacial prostheses. Int J Prosthodont. 1995;8(1):79-86.

12. CIE Technical report: colorimetry. Vienna: CIE Central Bureau; 2004. (CIE Publication, Vol 15.3).

13. Miletic V, Jakovljevic N, Manojlovic D, Marjanovic J, Rosic AA, Dramićanin MD. Refractive indices of unfilled resin mixtures and cured composites related to color and translucency of conventional and low-shrinkage composites. J Biomed Mater Res B Appl Biomater. 2017;105(1):7-13. https://doi.org/10.1002/ibm.b.33523

14. Drelich J. Nanoparticles in a liquid: new state of liquid? J Nanomater Mol Nanotechnol. 2013;2(1):1-2. https://doi.org/10.4172/2324-8777.1000e105 
15. Patel MP, Davy KW, Braden M. Refractive index and molar refraction of methacrylate monomers and polymers. Biomaterials. 1992;13(9):643-5. https://doi.org/10.1016/0142-9612(92)90034-L

16. Ferracane JL. Hygroscopic and hydrolytic effects in dental polymer networks. Dent Mater. 2006;22(3):211-22. https://doi.org/10.1016/i.dental.2005.05.005

17. Ruyter IE, Nilner K, Moller B. Color stability of dental composite resin materials for crown and bridge veneers. Dent Mater. 1987;3(5):246-51. https://doi.org/10.1016/S0109-5641(87)80081-7

18. Ghinea R, Perez MM, Herrera LJ, Rivas MJ, Yebra A, Paravina RD. Color difference thresholds in dental ceramics. J Dent. 2010;38(Suppl 2):e57-64. doi:

19. Paravina RD, Ghinea R, Herrera LJ, Bona AD, Igiel $C$, Linninger $M$ et al. Color difference thresholds in dentistry. J Esthet Restor Dent. 2015;27 Suppl 1:S1-9. https://doi.org/10.1111/jerd.12149
20. Pecho OE, Ghinea R, Perez MM, Della Bona A. Influence of gender on visual shade matching in dentistry. J Esthet Restor Dent. 2017;29(2):E15-23. https://doi.org/10.1111/jerd.12292

21. Fontes ST, Fernández MR, de Moura CM, Meireles SS. Color stability of a nanofill composite: effect of different immersion media. J Appl Oral Sci. 2009;17(5):388-91. https://doi.org/10.1590/S1678-77572009000500007

22. Meireles SS, Santos IS, Bona AD, Demarco FF. A double-blind randomized clinical trial of two carbamide peroxide tooth bleaching agents: 2-year follow-up. J Dent. 2010;38(12):956-63. https://doi.org/10.1016/i.jdent.2010.08.003

23. Hernandes DK, Arrais CA, Lima E, Cesar PF, Rodrigues JA. Influence of resin cement shade on the color and translucency of ceramic veneers. J Appl Oral Sci. 2016;24(4):391-6. https://doi.org/10.1590/1678-775720150550

24. Barutcigil Ç, Yıldız M. Intrinsic and extrinsic discoloration of dimethacrylate and silorane based composites. J Dent. 2012;40 Suppl 1:e57-63. https://doi.org/10.1016/i.jdent.2011.12.017 